\title{
Simulated Annealing e Hill Climbing Aplicado ao Problema de Programação de Horários do CCA-UFES
}

\author{
André Soares Carvalhd" \\ Departamento de Computação, Centro de Ciências Agrárias, UFES, Alegre, ES \\ Gelinton Pablo Mariand \\ Departamento de Computação, Centro de Ciências Agrárias, UFES, Alegre, ES \\ Edmar Hell Kampke $\beta^{3}$ \\ Departamento de Computação, Centro de Ciências Agrárias, UFES, Alegre, ES \\ Geraldo Regis Mauri. \\ Departamento de Computação, Centro de Ciências Agrárias, UFES, Alegre, ES
}

Resumo. Este trabalho tem como objetivo a aplicação de um método baseado na metaheurística Simulated Annealing (SA) para resolução do Problema de Programação de Horários em Universidades (PPHU). Após a meta-heurística, é usada a técnica de busca local denominada Hill Climbing (HC). Com o intuito de avaliar o desempenho do método proposto, foram utilizados os dados referentes à oferta de disciplinas do CCA-UFES realizada no período letivo 2013/2. Os resultados obtidos foram satisfatórios, auxiliando no processo de tomada de decisão ao apresentar boas soluções de forma rápida.

Palavras-chave. Problema de Programação de Horários em Universidades, Simulated Annealing, Hill Climbing

\section{Introdução}

Os Problemas de Programação de Horários em Universidades (PPHU) são problemas clássicos de otimização combinatória. O problema consiste em fixar uma sequência de encontros entre professores e alunos em um período prefixado de tempo satisfazendo a um conjunto de restrições de vários tipos. Em outras palavras, o objetivo é alocar um conjunto de aulas em um número pré-determinado de horários, satisfazendo diversas restrições que envolvem professores, alunos, currículos dos cursos e o espaço físico disponível [3].

As restrições do problema podem ser classificadas em restrições fortes, que devem ser satisfeitas a qualquer custo, e restrições fracas, cujo atendimento é desejável.

\footnotetext{
${ }^{1}$ andresoarescarvalho@gmail.com

${ }^{2}$ pablo06@gmail.com

3 edmar.kampke@ufes.br

${ }^{4}$ geraldo.mauri@ufes.br
} 
A modelagem do problema e as restrições usadas variam muito entre as instituições de ensino, o que dificulta a comparação de resultados. No caso específico do Centro de Ciências Agrárias (CCA) da Universidade Federal do Espírito Santo (UFES), onde existem 17 cursos de graduação de diferentes áreas, existem restrições específicas para esta realidade, sendo considerado neste trabalho 15 restrições $(7$ restrições fortes e 8 restrições fracas). Em um PPHU quanto maior o número de restrições mais difícil é encontrar uma solução que atenda todos os anseios das partes envolvidas. Assim, o PPHU do CCA-UFES pode ser considerado um problema difícil.

Para mensurar a qualidade das soluções do método proposto, foi utilizado uma função objetivo a ser minimizada, que foi modelada como $f(s)=\sum_{i=1}^{15} \delta_{i} R_{i}$, sendo que $s$ é a solução, $R_{i}$ é o número de violações da $i$-ésima restrição e $\delta_{i}$ é a penalização utilizada.

\section{Método Proposto}

Neste trabalho, foi utilizada a meta-heurística Simulated Annealing [2] para o PPHU do CCA-UFES. O método constrói uma solução inicial de forma gulosa, alocando as aulas mais difíceis primeiro. Em seguida, é gerada iterativamente uma solução vizinha por meio de movimentos de troca das aulas alocadas na solução original.

Em seguida, para intensificar a busca por boas soluções, foi utilizada a técnica de busca local denominada Hill Climbing (HC), originalmente proposta por Glover [1] para um problema de maximização. Essa técnica gera iterativamente uma solução vizinha que é avaliada. O processo termina após um número de iterações melhoras.

\section{Resultados e Conclusões}

Todos os parâmetros do problema foram calibrados empiricamente. O algoritmo proposto foi executado 10 vezes, com tempo máximo de execução definido em 500 segundos, em um computador com processador Core i7-3610 QM de 2.30 Ghz com 8GB de memória RAM utilizando Windows 7 de 64 bits.

O algoritmo proposto sempre encontrou soluções viáveis. O valor médio da função objetivo foi $f_{S A+H C}=511$, o que representa uma diminuição média de $32.8 \%$ em relação a tabela-horário construída pelos coordenadores de curso $\left(f_{\text {manual }}=760\right)$. Dessa forma, o algoritmo proposto foi satisfatório, permitindo que uma tomada de decisões seja definida de forma mais rápida e de melhor qualidade.

\section{Referências}

[1] F. Glover, Tabu Search-Part I, ORSA Journal of Computing, 190-206, (1989).

[2] S., Kirkpatrick, D. C. Gellat, M. P. Vecchi, Optimization by simulated annealing, Science, v. 220, 671-680, (1983).

[3] A. Schaerf, A survey of automated timetabling, Artificial Intelligence Review, v. 13, 87-127, (1999). 